\title{
Osteopontin improves adhesion and migration of human primary renal cortical epithelial cells during wound healing
}

\author{
JINFENG WU and ZUOLIN WANG \\ Laboratory of Oral Biomedical Science and Translational Medicine, School of Stomatology, \\ Tongji University, Shanghai 200072, P.R. China
}

Received April 22,2015; Accepted September 12, 2016

DOI: $10.3892 / \mathrm{ol} .2016 .5219$

\begin{abstract}
The aim of the present study was to investigate the effect of osteopontin (OPN) on adhesion and migration in human primary renal cortical epithelial cells during wound healing and Transwell assays. MTT assay was used to examine the cell viability and western blot analysis was used to examine the expression of cytoskeletal proteins and cell adhesion molecules. The results showed that overexpression of OPN had positive effects on the viability, proliferation, adhesion and migration of the human primary renal cortical epithelial cells. In addition, the integrity of the cell membrane and cytoskeleton of the epithelial cells was negatively affected by knockdown of OPN expression. The Transwell migration and a wound healing assays performed using OPN-knockdown cells suggested that OPN had a significant impact on cell migration $(\mathrm{P}=0.0421)$ and wound healing $(\mathrm{P}=0.0333)$. Therefore, OPN may be a potential target for the therapeutic modulation of skin repair to improve the healing rate and quality of wound healing.
\end{abstract}

\section{Introduction}

A complicated process is involved in wound healing, including inflammatory, proliferative and remodeling phases (1). In the initial inflammatory phase, the most distinguishing features are the infiltration of neutrophils and macrophages, along with hemostasis. The aim of the proliferative phase is to increase the density of scaffold for cell migration subsequent to debridement (2). In the remodeling phase, the process is induced by various growth factors or signaling molecules, including

Correspondence to: Professor Zuolin Wang, Laboratory of Oral Biomedical Science and Translational Medicine, School of Stomatology, Tongji University, 399 Middle Yanchang Road, Shanghai 200072, P.R. China

E-mail: zl_wang2014@yeah.net

Abbreviations: OPN, osteopontin; FAK, focal adhesion kinase

Key words: osteopontin, adhesion, migration epidermal growth factor, transforming growth factor- $\alpha$, platelet-derived growth factor, acidic fibroblast growth factor (FGF), basic FGF, keratinocyte growth factor, interleukin-1 and insulin-like growth factor, which play critical roles in the pro-fibrogenic and pro-inflammatory reactions (3). Previous studies have shown that osteopontin (OPN) participates in several diverse physiological and pathological processes, including wound healing, bone remodeling, cancer metastasis and invasiveness, and inhibition of apoptosis (3-6). OPN is a secreted phospho-protein that is synthesized by various immune cells, such as macrophages, dendritic cells and natural killer cells $(7,8)$. OPN activates cell signaling pathways by phosphorylation binding to extracellular receptors, including focal adhesion kinase (FAK), paxillin, tensin and $\operatorname{Src}(9,10)$. Previous studies have demonstrated that OPN is critical during the migration and proliferation of both human lung fibroblasts and mouse epithelial cells (11-13). However, whether OPN has an evident role in improving the ability of cell adhesion and migration in the deep layers of the wound remains unknown. The mechanism of action also remains unclear.

In the present study, the effect of knockdown or overexpression of OPN on human primary renal cortical epithelial cells is reported. The present study aimed to investigate the role of OPN in wound healing through its effects on human deep layers of epithelial cell migration, proliferation and adhesion. It was hypothesizes that OPN promotes epithelial cell migration, proliferation by increasing protein expression associated with cell adhesion.

\section{Materials and methods}

Reagents and cell culture. Antibodies against OPN (rabbit polyclonal anti-human; 1:1,500; ab8448), phosphorylated (p)-Y118-paxillin (rabbit polyclonal anti-human; 1:1,000; 2541), GAPDH [rabbit polyclonal anti-human; XP ${ }^{\circledR}$ Rabbit mAb (HRP Conjugate); 1:2,000; 8884), paxillin (rabbit anti-human; 1:1,000; 2542), FAK (rabbit polyclonal anti-human; 1:1,000; 3285) and pY Tyr576/577-FAK (rabbit polyclonal anti-human; $1: 1,000 ; 3281)$ were purchased from Cell Signaling Technology, Inc. (Danvers, MA, USA). Dulbecco's modified Eagle's medium (DMEM) was purchased from Gibco (Thermo Fisher Scientific, Inc., Waltham, MA, USA). Fetal bovine serum (FBS) was purchased from HyClone (GE Healthcare Life Sciences, Logan, UT, USA). All other reagents were obtained 
from Sigma-Aldrich (Merck Millipore, Darmstadt, Germany) unless otherwise described. The human primary renal cortical epithelial cells (Normal; PCS-400-011) were obtained from American Type Culture Collection (Manassas, VA, USA). The cells were maintained in DMEM containing $200 \mathrm{U} / \mathrm{ml}$ penicillin G (Gibco; Thermo Fisher Scientific, Inc.), $200 \mu \mathrm{g} / \mathrm{ml}$ streptomycin and 10\% FBS (HyClone; GE Healthcare Life Sciences) at $37^{\circ} \mathrm{C}$ in a humid atmosphere of $5 \% \mathrm{CO}_{2}$ at $37^{\circ} \mathrm{C}$.

Small interfering RNA (siRNA) and overexpression vector. siRNAs were synthesized by Shanghai GenePharma Co., Ltd. (Shanghai, China). The siRNA sequences for mouse OPN siRNA were as follows: siRNA sense, 5'-GAGGAGCCA UUUAUUGAAACUCG-3' and antisense, 5'-CGAGUUUCA AUAAAUGGCUCCUC-3'; and siRNA control (oligos with no matching GeneBank sequence) sense, 5'-GCGACGAUC UGCCUAAGAU-3' and antisense, 5'-AUCUUAGGCAGA UCGUCGC-3'. The pairs of siRNA oligonucleotides were prepared as a $20 \mu \mathrm{M}$ stock. The OPN gene was cloned into the pcDNA ${ }^{\mathrm{TM}} 3.1$ (-) Mammalian Expression Vector (V79520; Invitrogen; Thermo Fisher Scientific, Inc.) as the overexpression cassette.

For transient transfection, five groups were created, consisting of the control, overexpression group transfected with pcDNA3.1-OPN-GFP (over OPN), empty carrier group with overexpression of a blank plasmid (over blank), siRNA interference group (si-OPN) and siRNA blank (si-blank). The primary renal cortical endothelial cell line was cultured in 6-well plates to $80 \%$ confluence and then transfected using Lipofectamine RNAiMAX (Invitrogen; Thermo Fisher Scientific, Inc.) according to the manufacturer's instructions. The cells were collected $24 \mathrm{~h}$ subsequent to transfection for other experiments.

Cell viability assay. Cell viability was determined using the MTT method (14). The cells were plated into 96-well microtiter plates for $24 \mathrm{~h}$. Subsequently, $10 \mu \mathrm{l}$ of MTT-labeling reagent was added to each well and the plates were incubated at $37^{\circ} \mathrm{C}$ for $4 \mathrm{~h}$. The cultures were then solubilized and spectrophotometric absorbance of the samples was detected by a microtiter plate reader. The wavelength used to measure the absorbance of the formazan product was $570 \mathrm{~nm}$, with a reference wavelength of $750 \mathrm{~nm}$. Cell viability was expressed as a percentage of the positive control.

Wound healing assay. Wound healing experiments were performed for quantitative investigation of the migration of cells. Cells were grouped as aforementioned, consisting of the control, over OPN, over blank, si-OPN and si-blank groups. The initial cell seeding density was $\sim 1 \times 10^{5}$ cells $/ \mathrm{cm}^{2}$. A square wound was made using a cell scraper. The cells were washed twice with PBS and then incubated with fresh DMEM for another $24 \mathrm{~h}$ at $37^{\circ} \mathrm{C}$. The cells were then visualized by microscopy (IX71; Olympus, Corporation, Tokyo, Japan).

Transwell assay. A further cell migration identification was performed using Transwell assay. The cultured cells were detached with trypsin subsequent to $8 \mathrm{~h}$, and the treated cells were seeded into a Millicell (Greiner Bio-One, Frickenhausen, Germany) containing pores of $8 \mathrm{~mm}$ in diameter at an initial seeding density of $1 \times 10^{5}$ cells $/ \mathrm{cm}^{2}$, suspended in DMEM containing $1 \%$ fetal bovine serum. The Millicell pores were then put into the wells of a 24 -well plate containing $20 \%$ fetal bovine serum in DMEM. The cells on the Millicell pores were transfected with four types of vectors (over OPN, over blank, si-OPN and si-blank). Fresh DMEM was added $4 \mathrm{~h}$ later, and the cells were kept at $37^{\circ} \mathrm{C}$ for another $24 \mathrm{~h}$. The migrated cells were stained with crystal violet $(0.1 \% \mathrm{wt})$ and the number of migratory cells was recorded using an optical microscope at x100 magnification. The cell number was randomly counted in 12 different fields of view for each group, and the data were averaged from three parallel experiments.

$R N A$ extraction and reverse transcription-quantitative polymerase chain reaction $(R T-q P C R)$. Total RNA was isolated using TRIzol reagent (Invitrogen; Thermo Fisher Scientific, Inc.). Subsequent to being treated with RNase-free DNase I (Roche Applied Science, Penzberg, Germany), first-strand cDNA was synthesized with Moloney murine leukemia virus (M-MLV) reverse transcriptase and Oligo-dTs (Takara Bio, Inc., Otsu, Japan). RT-qPCR was conducted using the ABI Prism 7500 Sequence Detection System, according to the manufacturer's instructions (Applied Biosystems; Thermo Fisher Scientific, Inc.). Specific primers used for RT-qPCR were as follows: OPN forward, 5'-GAAGTTTCGCAGACC TGACAT-3' and reverse, 5'-GTATGCACCATTCAACTC CTCG-3'; FAK forward, 5'-AGTGGACCAGGAAATTGC TTTG-3' and reverse, 5'-GTGTTTTGGCCTTGACAGAAT CPCR-3'; paxillin forward, CTGCTGGAACTGAACGCT GTA-3' and reverse, 5'-GGGGCTGTTAGTCTCTGGGA-3'; GAPDH forward, 5'-ACAACTTTGGTATCGTGGAAGG-3' and reverse, 5'-GCCATCACGCCACAGTTTC-3'. For the relative comparison of each gene, we analyzed the $\mathrm{Cq}$ value of qPCR data with the $2^{-\Delta \Delta C q}$ method normalized by the endogenous control GAPDH (15).

Sample preparation and western blotting. The procedure for immunoblotting has been previously described (16). Cells were treated with cell lysis buffer and then protein samples were collected. Briefly, $40 \mu \mathrm{g}$ protein/lane was loaded into the lanes of $12 \%$ denatured polyacrylamide gel. The proteins were separated by electrophoresis and transferred to nitrocellulose membranes $(0.45 \mu \mathrm{m}$; Schleicher \& Schuell; GE Healthcare Life Sciences). The membranes were blocked with $3 \%$ bovine serum albumin in $0.01 \mathrm{M}$ PBS ( $\mathrm{pH} 7.4$ ) and $0.05 \%$ Tween-20 (PBST) at room temperature for $1 \mathrm{~h}$. Subsequently, the membrane was incubated with the aforementioned primary antibodies against the target proteins overnight at $4^{\circ} \mathrm{C}$. Following two quick washes in PBST, the membranes were incubated with secondary antibodies conjugated with horseradish peroxidase [Goat Anti-Rabbit IgG H\&L (HRP); dilution, 1:4,000 in PBST; ab97051; Abcam, Cambridge, UK) for $2 \mathrm{~h}$ at room temperature. Following washes as aforementioned, an enhanced chemiluminescence reagent $\left(\right.$ Pierce $^{\mathrm{TM}}$ DAB Substrate kit; 34002; Thermo Fisher Scientific, Inc.) was used to produce fluorescence for detection. The density of immunoblotting was quantified using Quantity One software (Bio-Rad Laboratories, Inc., Hercules, CA, USA). The relative expression of the target protein was quantified as a ratio against GAPDH. 

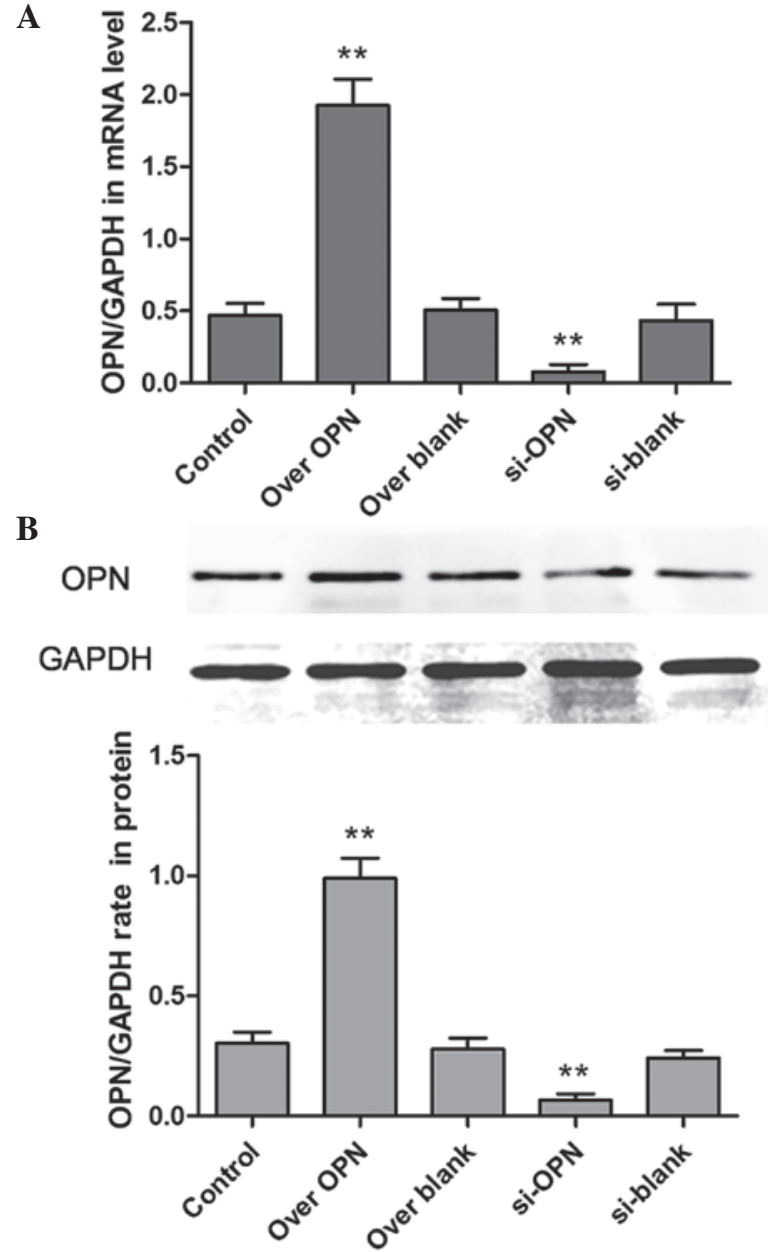

Figure 1. Efficiency of the up- or downregulation of OPN. Five groups, consisting of the control, over OPN, over blank, si-OPN and si-blank groups, were formed based on the transient transfection of the human primary renal cortical endothelial cell line. The expression of OPN was detected by (A) reverse transcription-polymerase chain reaction and (B) western blot analysis. ${ }^{* *} \mathrm{P}<0.01$. OPN, osteopontin; over OPN, over-expression of pcDNA3.1-OPN-green fluorescent protein; over blank, empty carrier over-expression; si-OPN, small interfering RNA interference; si-blank, small interfering RNA.

Statistical analysis. Experiments were performed routinely with $>4$ samples per group, with the values expressed as the mean \pm standard error of the mean. All experiments were replicated at least three times. Statistical analysis was performed using one-way analysis of variance and Student's t-test for multiple comparisons. $\mathrm{P}<0.05$ was considered to indicate a statistically significant difference.

\section{Results}

Efficiency of up- or downregulation of OPN expression. The OPN expression was assessed by analyzing the protein and mRNA levels. The results showed that the overexpression vector was evidently effective, while the siRNA, which was selected from several alternative siRNA sequences, could efficiently disturb the expression of OPN (Fig. 1). The expression of the over OPN group was significantly increased compared with the blank group ( $\mathrm{P}=0.0011)$. In addition, the expression of si-OPN group was significantly decreased compared with the blank $(\mathrm{P}=0.0021)$.
A

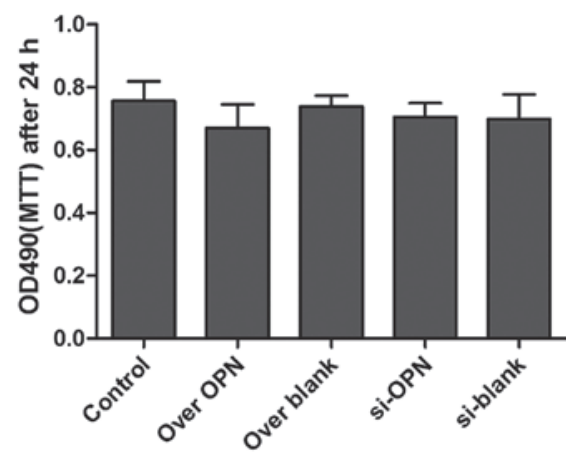

B

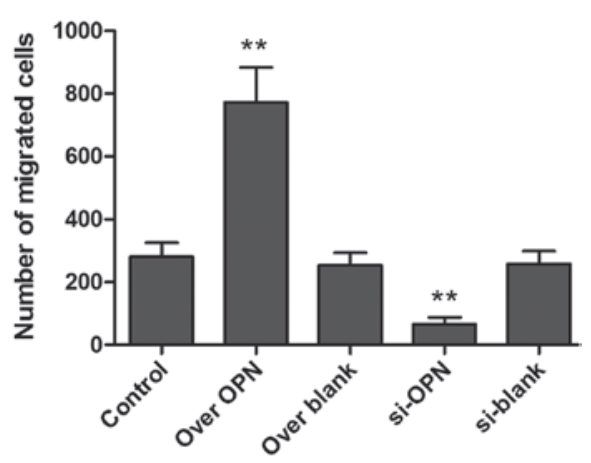

Figure 2. Determination of proliferation or migration of the human primary renal cortical endothelial cell using MTT and Transwell assays. (A) The value of MTT in the control, over OPN, over blank, si-OPN and si-blank groups. (B) Statistical analysis of migrated cells in the Transwell. All the data are presented as the mean \pm standard error of the mean of 4 individual experiments. ${ }^{* *} \mathrm{P}<0.01$. OPN, osteopontin; over OPN, over-expression of pcDNA3.1-OPN-green fluorescent protein; over blank, empty carrier over-expression; si-OPN, small interfering RNA interference; si-blank, small interfering RNA.

Effects of OPN on cell proliferation. To investigate the effects of OPN on the proliferation of human epithelial cells, the MTT assay was used to examine the cell viability. However, there was no significant difference between cell viability in the si-OPN group, over OPN group and the control groups (Fig. 2). The results showed that increasing or decreasing OPN expression did not significantly affect the cell proliferation.

Overexpression of OPN increases human epithelial cell migration. Macroscopic analyses of time-matched OPN overexpression vs. control wounds showed that closure was markedly accelerated $(\mathrm{P}=0.0372$ and $\mathrm{P}=0.0241$, respectively) at early time points (12 and $18 \mathrm{~h}$ ). Cells transfected with OPNoverexpressing vectors exhibited a mean closure of the wound of $56 \%$ after $24 \mathrm{~h}$, while cells transfected with OPN-siRNA vectors achieved a mean closure of the wound of $12 \%$ by $24 \mathrm{~h}$, compared with the $24 \%$ average closure observed in the blank control (Fig. 3A). Since OPN-overexpressing cells are capable of almost complete and efficient wound closure, OPN may play a pivotal role in the repair of deep layers of skin.

Transwell assay was also used to detect the migration of epithelial cells. The number of migrated cells were counted, and their shape is shown in Fig. 3B. The numbers of migrated cells in the over-OPN and si-OPN groups were significantly increased $(\mathrm{P}=0.0223)$ and decreased $(\mathrm{P}=0.0421)$, respectively, compared with the three blank groups, indicating that OPN plays a vital role in accelerating cell migration. 
A
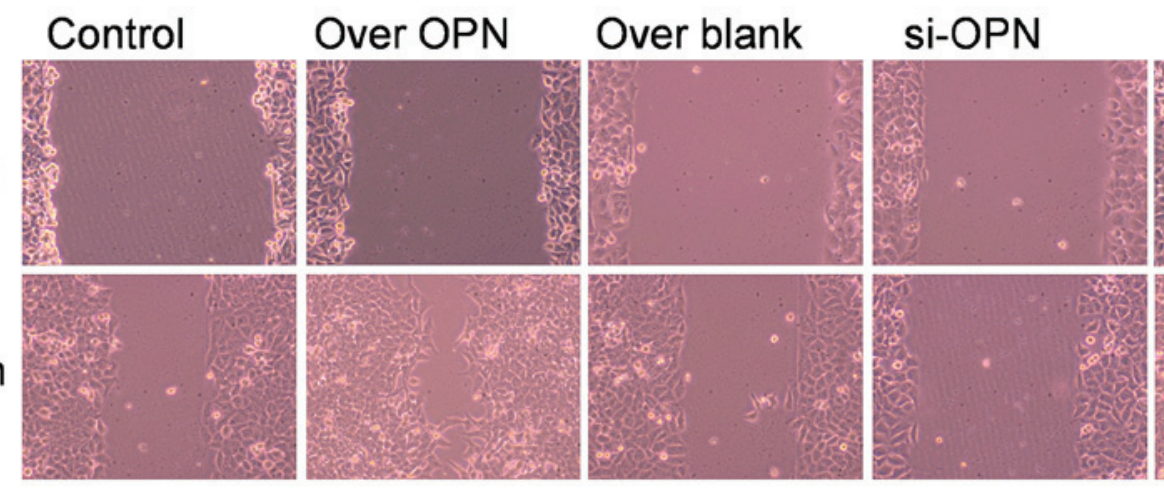

si-blank

$24 \mathrm{~h}$
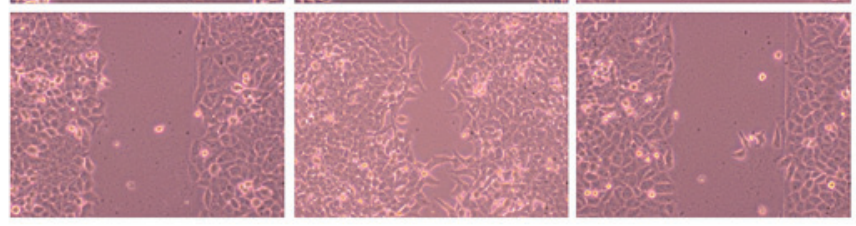

B
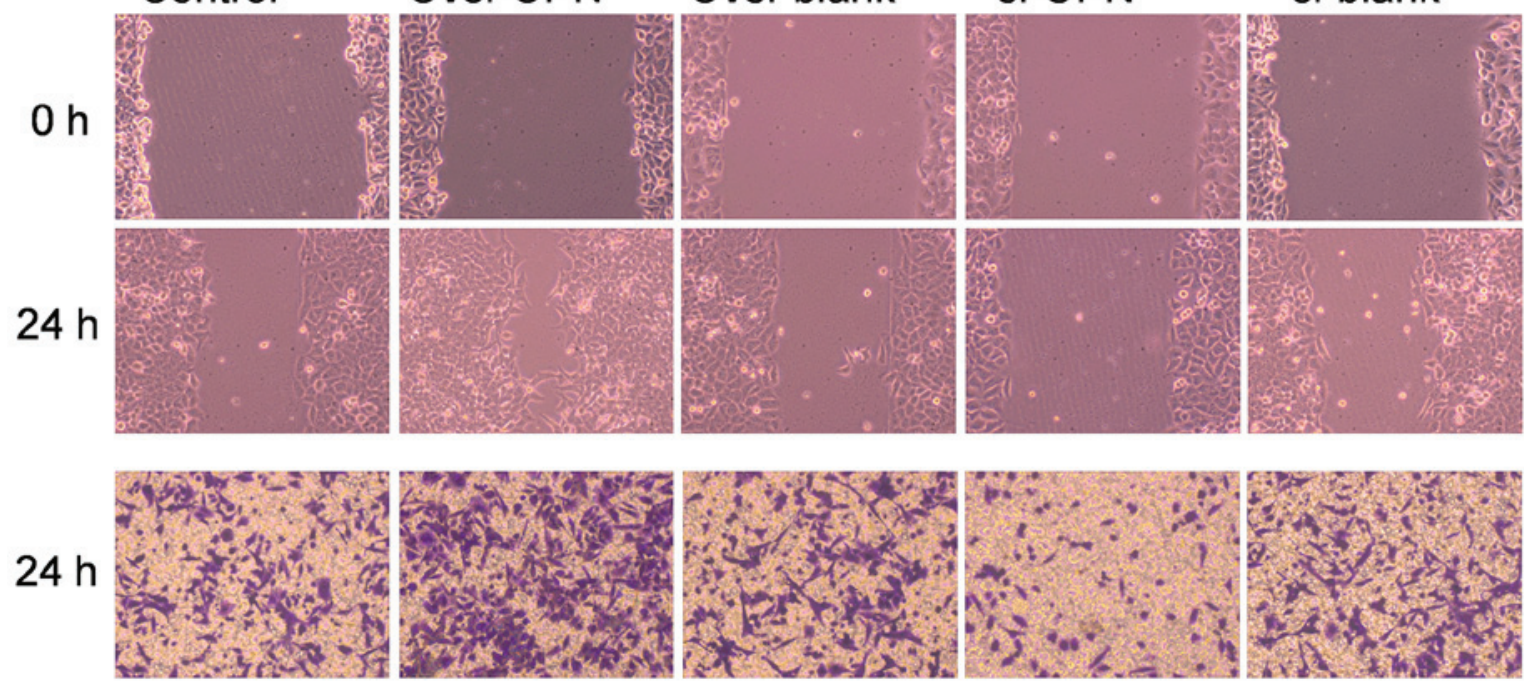

Figure 3. Effect of OPN on cell migration in the human primary renal cortical endothelial cell line. (A) Wound healing assay was used to examine cell migration. Cells with high OPN expression exhibited stronger ability of healing, since the migrated cell number was significantly higher than that of the control $(\mathrm{P}=0.0132)$. OPN-knockdown cells had a lower migrating ability than the control $(\mathrm{P}=0.0333)$. (B) Cell invasion was examined by Transwell assay. Cells with high OPN expression displayed stronger invasive ability, since the stained cell number was significantly higher than that of the control $(\mathrm{P}=0.0223)$. $\mathrm{Cells}$ with low OPN expression (treated by OPN siRNA) displayed a lower invasive ability, and the number of stained cells was significantly lower than that of the control ( $\mathrm{P}=0.0421)$. Magnification, x100. OPN, osteopontin; over OPN, over-expression of pcDNA3.1-OPN-green fluorescent protein; over blank, empty carrier over-expression; si-OPN, small interfering RNA interference; si-blank, small interfering RNA.

A

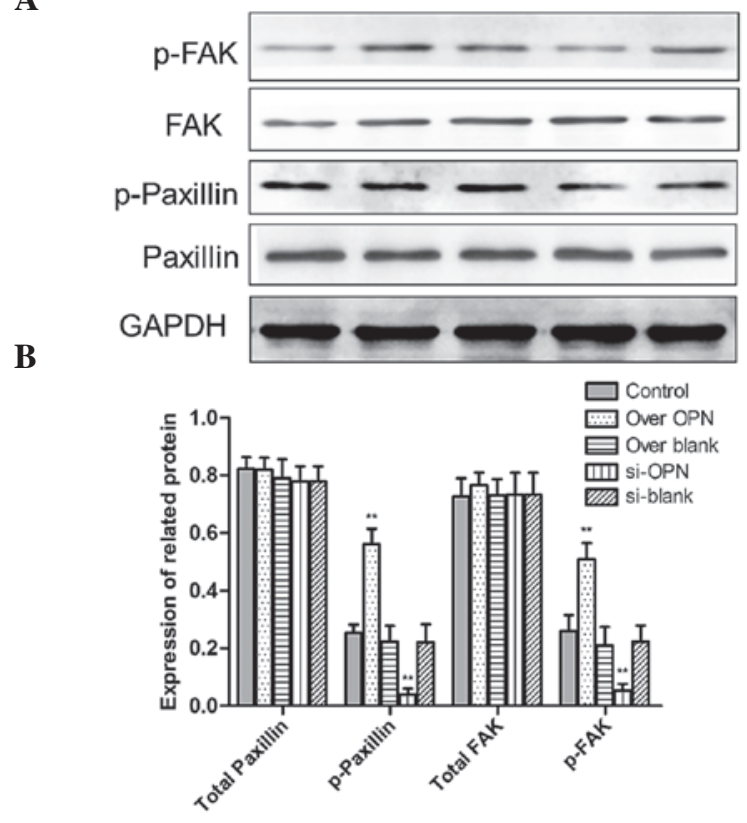

Figure 4. Effect of OPN knockdown or over-expression on adhesion protein and GAPDH expression in the human primary renal cortical endothelial cell line. (A) The expression of pY118-paxillin, pY-FAK, total paxillin and total FAK was examined by western blot analysis. (B) Statistical analysis of protein expression from western blotting. All data are presented as the mean \pm standard error of the mean of 4 individual experiments. ${ }^{* *} \mathrm{P}<0.01$. OPN, osteopontin; FAK, focal adhesion kinase; over OPN, over-expression of pcDNA3.1-OPN-green fluorescent protein; over blank, empty carrier over-expression; si-OPN, small interfering RNA interference; si-blank, small interfering RNA.

High expression of OPN improves the adhesion of human epithelial cells. To investigate whether OPN induced stronger cell attachment to the substrate, the expression of cell adhesion molecules, including FAK and paxillin were also examined by western blotting. The overexpression of OPN improved the phosphorylation of paxillin $(\mathrm{P}=0.0001)$ and FAK $(\mathrm{P}=0.0001)$. Additionally, protein expression of pY118-paxillin $(\mathrm{P}=0.0012)$ and pY-FAK $(\mathrm{P}=0.0002)$ was inhibited by OPN downregulation. However, there is no difference in the total FAK or paxillin among the five groups (Fig. 4). This result shows that the ability of cell adhesion is decreased by OPN, while OPN is involved in modulating signaling molecules associated with adhesion during healing of the deep layers of a wound.

\section{Discussion}

OPN is an inflammation-dependent gene that is expressed in wound granulation tissue accompanied by the inflammatory response (17). OPN is involved in signal transduction as a secreted chemotactic factor $(18,19)$, depending on the cellular microenvironment. In addition, OPN may regulate cell adhesion, migration, survival and immune reactions, as well as the balance between osteoblasts and osteoclasts (10). It has been reported that OPN-knockout mice developed normally, but the healing of skin wounds had a longer time course compared with wild-type mice. Notably, there was a large alteration in matrix architecture and collagen fibril formation in the deep layers of the wound (13). However, the mechanism of this alteration remains unclear.

OPNcombined withcellreceptors, particularly $\alpha v \beta 3$-integrin, was considered responsible for cell adhesion, migration, proliferation and cytoskeletal regulation by phosphorylation of FAK, paxillin, tensin or Src, which activate downstream signal pathways in specific cellular responses $(20,21)$. However, the mechanism of OPN increasing the cell contractility and adhesion remains unknown, since OPN binding to $\alpha v \beta 3$-integrin may change the cytoskeletal organization by activating FAK (20). In 
the present study, the expression of pY118-paxillin and pY-FAK was significantly increased by OPN overexpression during deep layers of wound healing.

These results provide insight into the role of OPN in the deep layer of human epithelial cells during wound healing. Previous studies have demonstrated that OPN plays an important role in the occurrence and development of hepatic fibrosis by examining organ fibrosis $(12,22,23)$. Other studies have also shown that OPN may induce the migration and proliferation of lung fibroblasts in human idiopathic pulmonary fibrosis (11). Furthermore, the function of OPN in certain aspects of wound healing, such as the effect on human epithelial cells, remains unknown. The present study contributed to this knowledge by discussing the effects of OPN on cell adhesion and migration in human epithelial cells. The current study demonstrated that OPN strongly advanced the migration and adhesion of epithelial cells with OPN upregulation, similar effects are evidently inhibited by OPN downregulation, and the cell viability and proliferation were not affected by OPN expression.

Therefore, OPN is a key factor for promoting closure of healing wounds and improving cell adhesion in deep layers of skin.

\section{References}

1. Sen CK: Wound healing essentials: Let there be oxygen. Wound Repair Regen 17: 1-18, 2009.

2. Lee MY and Ehrlich HP: Influence of vanadate on migrating fibroblast orientation within a fibrin matrix. J Cell Physiol 217: 72-76, 2008

3. Miyazaki K, Okada Y, Yamanaka O, Kitano A, Ikeda K, Kon S, Uede T, Rittling SR, Denhardt DT, Kao WW and Saika S: Corneal wound healing in an osteopontin-deficient mouse. Invest Ophthalmol Vis Sci 49: 1367-1375, 2008.

4. Hunter C, Bond J, Kuo PC, Selim MA and Levinson H: The role of osteopontin and osteopontin aptamer (OPN-R3) in fibroblast activity. J Surg Res 176: 348-358, 2012.

5. McKee MD, Pedraza CE and Kaartinen MT: Osteopontin and wound healing in bone. Cells Tissues Organs 194: 313-319, 2011.

6. Miragliotta V, Pirone A, Donadio E, Abramo F, Ricciardi MP and Theoret CL: Osteopontin expression in healing wounds of horses and in human keloids. Equine Vet J 48: 72-77, 2016.

7. O'Regan A and Berman JS: Osteopontin: A key cytokine in cell-mediated and granulomatous inflammation. Int J Exp Pathol 81: 373-390, 2000.

8. Buback F, Renkl AC, Schulz G and Weiss JM: Osteopontin and the skin: Multiple emerging roles in cutaneous biology and pathology. Exp Dermatol 18: 750-759, 2009.
9. Mi Z, Guo H, Russell MB, Liu Y, Sullenger BA and Kuo PC: RNA aptamer blockade of osteopontin inhibits growth and metastasis of MDA-MB231 breast cancer cells. Mol Ther 17: 153-161, 2009.

10. Denhardt DT, Noda M, O'Regan AW, Pavlin D and Berman JS: Osteopontin as a means to cope with environmental insults: Regulation of inflammation, tissue remodeling, and cell survival. J Clin Invest 107: 1055-1061, 2001.

11. Pardo A, Gibson K, Cisneros J, Richards TJ, Yang Y, Becerril C, Yousem S, Herrera I, Ruiz V, Selman M and Kaminski N: Up-regulation and profibrotic role of osteopontin in human idiopathic pulmonary fibrosis. PLoS Med 2: e251, 2005.

12. Persy VP, Verhulst A, Ysebaert DK, De Greef KE and De Broe ME: Reduced postischemic macrophage infiltration and interstitial fibrosis in osteopontin knockout mice. Kidney Int 63: 543-553, 2003

13. Liaw L, Birk DE, Ballas CB, Whitsitt JS, Davidson JM and Hogan BL: Altered wound healing in mice lacking a functional osteopontin gene (spp1). J Clin Invest 101: 1468-1478, 1998.

14. Chen YY, Chen G, Fan Z, Luo J and Ke ZJ: GSK3beta and endoplasmic reticulum stress mediate rotenone-induced death of SK-N-MC neuroblastoma cells. Biochem Pharmacol 76: 128-138, 2008.

15. Yang G, Meng Y, Li W, Yong Y, Fan Z, Ding H, Wei Y, Luo J and Ke ZJ: Neuronal MCP-1 mediates microglia recruitment and neurodegeneration induced by the mild impairment of oxidative metabolism. Brain Pathol 21: 279-297, 2011.

16. Ke ZJ, DeGiorgio LA, Volpe BT and Gibson GE: Reversal of thiamine deficiency-induced neurodegeneration. J Neuropathol Exp Neurol 62: 195-207, 2003.

17. Leitner L, Jürets A, Itariu BK, Keck M, Prager G, Langer F, Grablowitz V, Zeyda M and Stulnig TM: Osteopontin promotes aromatase expression and estradiol production in human adipocytes. Breast Cancer Res Treat 154: 63-69, 2015

18. El-Tanani MK, Campbell FC, Kurisetty V, Jin D, McCann M and Rudland PS: The regulation and role of osteopontin in malignant transformation and cancer. Cytokine Growth Factor Rev 17: 463-474, 2006.

19. Shinohara ML, Lu L, Bu J, Werneck MB, Kobayashi KS, Glimcher LH and Cantor H: Osteopontin expression is essential for interferon-alpha production by plasmacytoid dendritic cells. Nat Immunol 7: 498-506, 2006.

20. Sodek J, Ganss B and McKee MD: Osteopontin. Crit Rev Oral Biol Med 11: 279-303, 2000.

21. Frisch SM, Vuori K, Ruoslahti E and Chan-Hui PY: Control of adhesion-dependent cell survival by focal adhesion kinase. J Cell Biol 134: 793-799, 1996.

22. Trueblood NA, Xie Z, Communal C, Sam F, Ngoy S, Liaw L, Jenkins AW, Wang J, Sawyer DB, Bing OH, et al: Exaggerated left ventricular dilation and reduced collagen deposition after myocardial infarction in mice lacking osteopontin. Circ Res 88: 1080-1087, 2001.

23. Matsui Y, Jia N, Okamoto H, Kon S, Onozuka H, Akino M, Liu L, Morimoto J, Rittling SR, Denhardt D, et al: Role of osteopontin in cardiac fibrosis and remodeling in angiotensin II-induced cardiac hypertrophy. Hypertension 43: 1195-1201, 2004. 